\title{
« Je ne connais pas Ben Sadok »
}

Quelques remarques sur le témoignage de Sartre au procès Ben Sadok

Jean Bourgault et Grégory Cormann

\section{(e) OpenEdition}

Journals

Édition électronique

URL : http://journals.openedition.org/genesis/1376

DOI : 10.4000/genesis. 1376

ISSN : 2268-1590

Éditeur :

Presses universitaires de Paris Sorbonne (PUPS), Société internationale de génétique artistique littéraire et scientifique (SIGALES)

\section{Édition imprimée}

Date de publication : 17 novembre 2014

Pagination : 57-70

ISBN : 9782840509714

ISSN : 1167-5101

\section{Référence électronique}

Jean Bourgault et Grégory Cormann, « « Je ne connais pas Ben Sadok » », Genesis [En ligne], 39 | 2014, mis en ligne le 12 décembre 2016, consulté le 20 avril 2019. URL : http://journals.openedition.org/ genesis/1376 ; DOI : 10.4000/genesis.1376 


\title{
« Je ne connais pas Ben Sadok. » Quelques remarques sur le témoignage de Sartre au procès Ben Sadok
}

\author{
Jean Bourgault et Grégory Cormann
}

$\mathrm{L}$ e 26 mai 1957, au stade de Colombes, alors que venait de s'achever la finale de la coupe de France de football, Mohamed Ben Sadok assassinait Ali Chekkal, ancien vice-président de l'Assemblée algérienne, qui avait assisté au match aux côtés du président de la République, René Coty 1 . Arrêté sur le champ, il fut incarcéré jusqu'à son procès, qui se tint du 9 au 11 décembre 1957 et eut un grand retentissement. Le 10 décembre - le jour même où Camus, à Stockholm, prononçait son discours de réception du prix Nobel -, maître Stibbe, qui assurait la défense de l'accusé, fit venir à la barre de nombreuses personnalités, dont Jean Rous, Émile Kahn, Germaine Tillion et Jean-Paul Sartre 2 .

Simone de Beauvoir a rapporté la façon dont Sartre vécut ce moment :

Sartre fut un des derniers à déposer. Rien ne marqua son trouble sinon que, parlant avec une déférence compassée du mort, il l'appela Ali Chacal. Comparant son attitude à celle de Ben Saddok (sic), il expliqua que les jeunes ne pouvaient pas consentir à la patience de leurs aînés car ils ne connaissaient de la France qu'un visage sanguinaire. Il souligna ensuite que l'acte accompli par Saddok était un meurtre politique et ne devait pas être assimilé à un attentat terroriste. Il faisait un gros effort pour parler un langage qui ne choquât pas le tribunal, et celui-ci parut soulagé par sa modération.

Ensuite déposèrent Massignon puis Germaine Tillion ; la France, constata-t-elle, avait acculé la jeunesse à la haine. Un instituteur avait proposé à ses élèves, des musulmans d'une dizaine d'années, ce sujet de rédaction : «Que feriez-vous si vous étiez invisibles ?»; elle lut quelques-unes de leurs compositions : tous avaient répondu, à travers des fantasmes divers : «Je tuerais tous les Français. »

\begin{abstract}
Je quittai la salle. Dans les couloirs, le général Tubert tonnait contre les Français d'Algérie. Tous les témoins louaient l'impartialité du président et la liberté qu'il leur avait accordée. Ils commentaient avec sévérité l'absence de Camus. Sa voix aurait eu d'autant plus de poids qu'on venait de lui décerner le prix Nobel. Stibbe lui avait seulement demandé de lire tout haut ce qu'il avait écrit dans un récent essai où il condamnait la peine de mort : il avait refusé de paraître à la barre et même d'envoyer un message au tribunal. Pour réclamer l'indulgence du juge, plusieurs témoins l'avaient cité, parfois non sans malice.

Je dînai à la Palette avec Sartre et Lanzmann. Saddok sauverait-il ou non sa tête ? Nous étions anxieux. Pour se consoler de la tension à laquelle toute la journée il avait été soumis, Sartre but du whisky : il supportait mal l'alcool depuis quelque temps et son agitation s'accrut : bientôt il tomba dans une morosité furieuse : «Dire que j'ai fait l'éloge de Chekkal ! Et j'ai parlé contre le terrorisme : comme si je condamnais le terrorisme ! Tout ça pour plaire aux poujadistes du jury ! Vous vous rendez compte ! » Le dépit, la rage lui mettaient les larmes aux yeux ${ }^{3}$.
\end{abstract}

1. Sur cet assassinat, on pourra lire Rachid Boudjedra, Le Vainqueur de coupe, Paris, Gallimard, 1989, ainsi que Les Figuiers de barbarie, Paris, Grasset, 2010.

2. Agnès Masson, conservatrice générale du Patrimoine et directrice des Archives nationales, nous a fait savoir que le témoignage de Sartre ne figure pas au dossier de procédure.

3. Simone de Beauvoir, La Force des choses, t. II, Paris, Gallimard, coll. «Folio», 1972 [1963], p. 141-142. Un point doit être corrigé : maître Stibbe avait sollicité Camus, qui écrivit une lettre au président de la Cour d'Assises de la Seine, en lui demandant qu'il fasse part au jury, mais sans rendre publique son intervention, qu'il estimait, tout en condamnant les actes de Ben Sadok, que la peine capitale serait à la fois inhumaine et désastreuse du point de vue politique (voir James D. Le Sueur, Uncivil War: Intellectuals and Identity Politics During the Decolonization of Algeria, Lincoln, University of Nebraska Press, 2005, p. 121). 
Les articles de presse du 11 décembre 1957 ne consacrèrent que peu de lignes aux déclarations des diverses personnalités appelées à témoigner la veille. Sartre fut présenté comme arrivant en «vedette américaine ». On signala qu'il avait insisté pour qu'on ne fasse pas l'amalgame entre assassinat politique et meurtre terroriste. Certains s'étonnèrent de la comparaison que fit Sartre entre Ben Sadok et Charlotte Corday. D'évidence, selon plusieurs récits, Ben Sadok écouta Sartre avec admiration. Le même jour, la cour d'assises de la Seine rendait son verdict : Ben Sadok échappait à la peine de mort et était condamné à la réclusion criminelle à perpétuité. Il serait plus tard gracié.

La Bibliothèque nationale de France conserve le manuscrit du texte rédigé par Sartre en vue de son témoignage 4 . Il porte les traces des tensions contradictoires dans lesquelles il a été écrit et constitue un document génétique très précieux. Il se compose de vingt-quatre feuillets, auxquels s'ajoute un feuillet dactylographié qui n'est pas de Sartre. Ce feuillet dactylographié constitue sans doute une sorte de fiche que la défense avait fait parvenir à chaque témoin, afin qu'il soit averti de l'axe selon lequel cette défense devait s'organiser. Ali Chekkal y est décrit comme le représentant « de la politique absurde menée par les gouvernements français en Algérie », Ben Sadok comme un jeune homme fin et cultivé, «symbole de la présence culturelle de la France en Algérie »; on y rapporte brièvement l'histoire de Ben Sadok : bon élève, il émigre en France après son service militaire et apprend le métier de plombier. Le texte décrit ensuite l'axe selon lequel il convient d'aborder l'acte commis :

Ce meurtre s'inscrit dans la lignée des grands meurtres politiques et ne relève ni du terrorisme aveugle, ni du règlement de compte. Cependant c'est sur lui que les ultras voudraient que l'on fasse un exemple en l'envoyant à l'échafaud : l'accusation va soutenir que sa condamnation à mort est nécessaire pour mettre fin au terrorisme et que toute indulgence serait une lâcheté envers les soldats français qui se battent en Algérie (f. 15).

Si l'on classe les feuillets manuscrits, on peut mettre au jour une campagne d'écriture rapide qui, tout en étant fidèle au programme proposé par la défense, témoigne aussi des difficultés éprouvées par Sartre à politiser la question sans la radicaliser. L'étude du document permet de dégager cinq ensembles de feuillets : trois esquisses préparatoires, un plan, et l'élaboration finale du texte 5 . Sartre commence d'emblée par rédiger un mouvement d'ouverture, afin, sans doute, de se disposer au vif de l'exposition orale et de faire l'épreuve, dans le mouvement de cette exposition, de l'orientation exigée par la défense. Ce n'est qu'au quatrième temps de rédaction que Sartre élabore un brouillon dont le cinquième temps suit scrupuleusement le dessein. Le texte final court sur cinq feuillets et compte un peu plus de neuf mille caractères.

Le premier temps n'occupe qu'un feuillet. L'ouverture est d'emblée polémique et politique : Sartre s'étonne de la présence, comme témoin de l'accusation, de Jacques Soustelle, qui défend avec feu l'Algérie française. Ce faisant, il choisit de dénoncer la politisation du procès et d'en appeler à la paix et aux valeurs de l'humanisme. Les premières lignes du manuscrit sont décisives :

On a parlé politique. Soustelle ne connaissait pas l'accusé : venu demander sa tête. Un homme de l'importance de Soustelle se dérange pour vous demander la tête d'un homme. Voilà la preuve que c'est politique. Il faut reprendre l'affaire sur un plan humain. La justice n'est pas la politique. Et ne doit pas l'être. Pas faire la guerre. C'est-à-dire qu'elle ne doit pas être répression ni intimidation (f. 13, fig. 1).

Deux oppositions s'entendent dans cette ouverture, qui s'articulent de façon approximative : l'opposition entre justice politique et justice humaine, d'une part,

4. Bibliothèque nationale de France, département des Manuscrits, fonds Sartre (NAF 28405), boîte « Articles et conférences ; 1944/1973 », souschemise violette portant l'indication «Ben Sadok / 24 f. / 1957 ». Seuls les trois premiers feuillets sont numérotés, d'une autre main que celle de Sartre. Des photographies des feuillets 1 et 2 de ce manuscrit figurent au catalogue de l'exposition «Sartre » de la BnF (Sartre, dir. Mauricette Berne, BnF/Gallimard, 2005, p. 222-223). Voir ici la reproduction du feuillet [13], fig. 1 .

5. L'ordre des feuillets doit être établi comme suit : première esquisse, début de rédaction : f. 13 ; deuxième esquisse : f. $1,4,7,5$, avec deux reprises, f. 14 et f. 6 ; troisième esquisse : f. 2,12 , avec deux reprises : f. $8,9,10,11$ et f. 3 ; élaboration du plan : f. 22, 23, 24, 25, 21 ; rédaction finale : f. 16, 17, 18, 19, 20. Le feuillet 15, dactylographié, n'est pas un texte de Sartre. 
«JE NE CONNAIS PAS BEN SADOK»

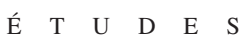

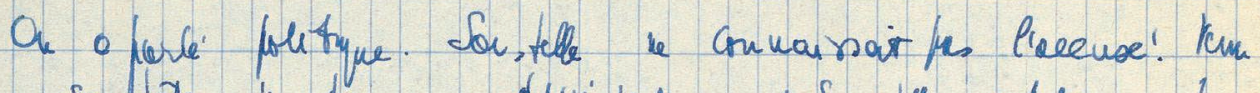

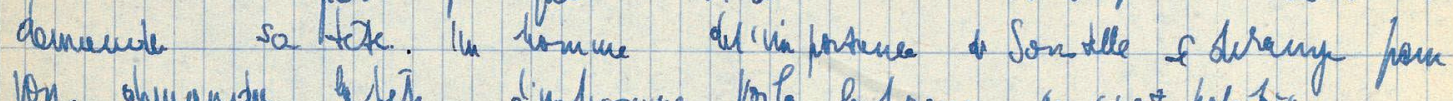

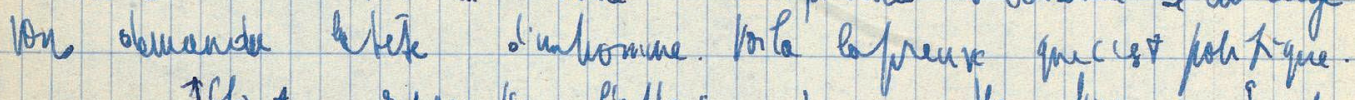

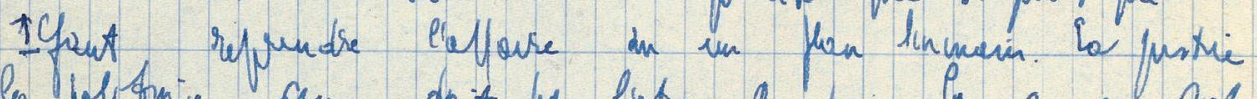

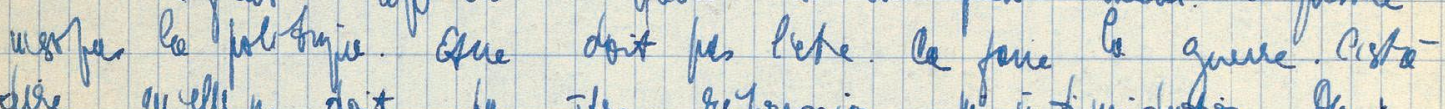

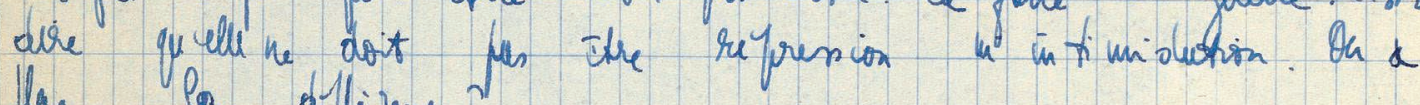
He la álience?

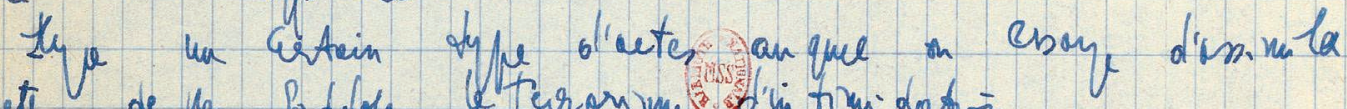

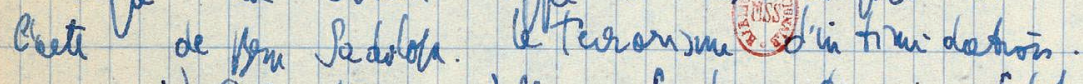

1) Carvetére : hllain, lovar, Oruneip. Solitude.

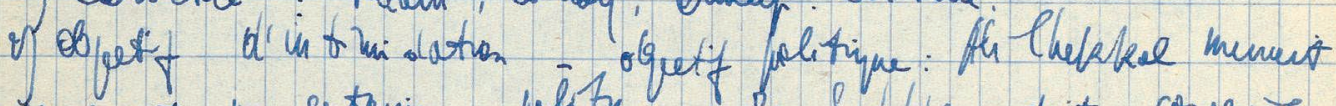

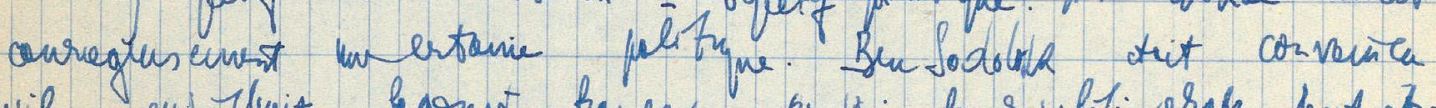

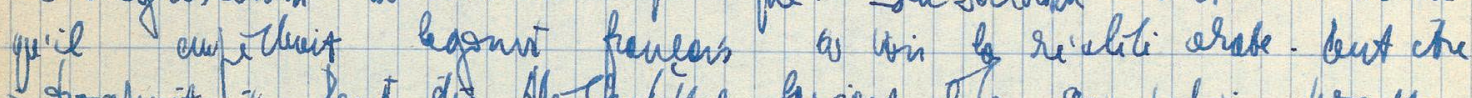

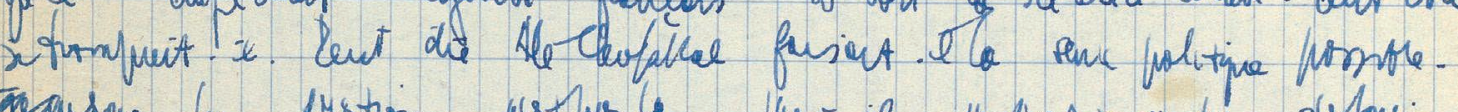

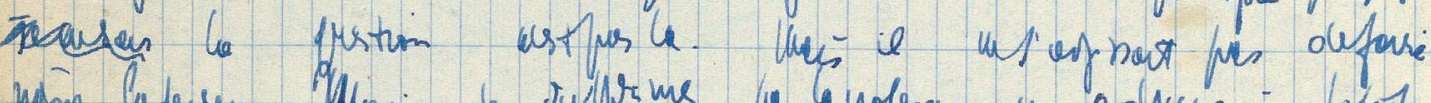

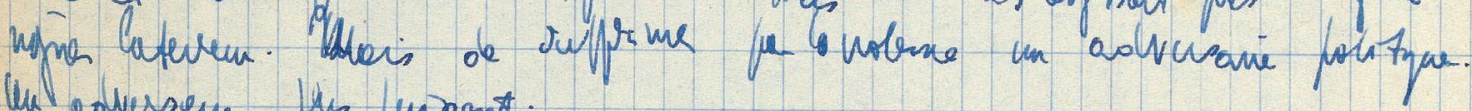
Ual edvuseve the jurront.

1) Conutain tholitude.

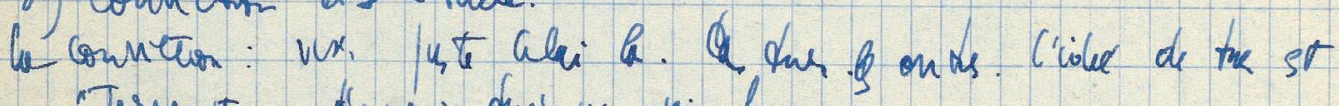

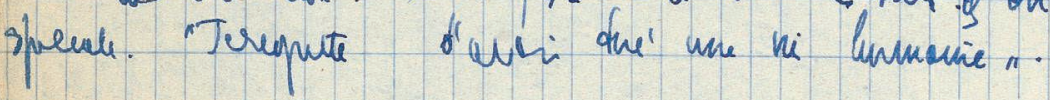

r'ir citate ba quil fut fuph. Ce un antur. Et

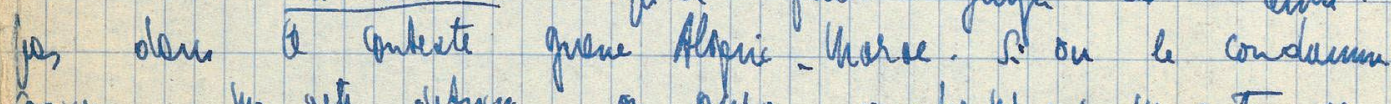

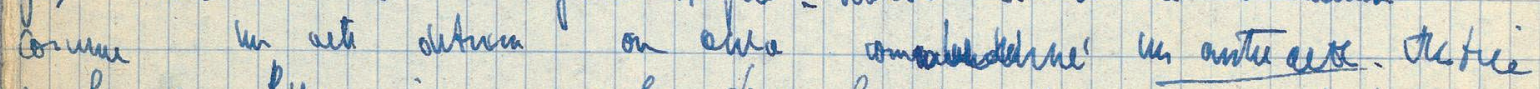

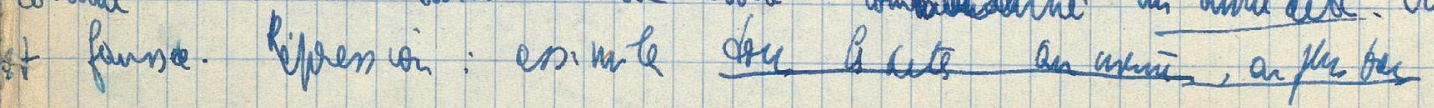

Fig. 1 : Jean-Paul Sartre, Dossier « Ben Sadok », f. [13] (BnF, NAF 28405)

59 
l'opposition entre acte terroriste et acte politique, d'autre part. La seconde fait entendre positivement le terme de « politique », en le distinguant de celui de «terroriste » (Ben Sadok a bien assassiné Chekkal, mais ses motifs sont politiques, et non terroristes), alors que la première dévalorise ce même terme de «politique » en l'associant à celui de « terrorisme » : une justice politique se soucierait en fait de continuer la guerre, par là serait partisane, violente, et liée au terrorisme : et il faut une justice humaine pour sortir de ce jeu infernal.

Étrange premier mouvement, décidément, qui voit Sartre se fonder sur 1'humanisme dont il est un des critiques les plus déterminés. Certes il n'ignore en rien, alors qu'il est en train d'écrire la Critique de la Raison dialectique, qu'une justice faite sur « le plan humain » ne peut être qu'une justice dissimulant des motifs politiques ; mais il s'agit ici de jouer la rhétorique de l'accusation contre elle-même, et pour cela de proposer une définition spécifique du «terrorisme »: il y a terrorisme lorsqu'il y a « répression », entendons : violence (et en ce sens, les dernières lignes de la version finale le diront : toute justice politique, répressive, a partie liée avec le terrorisme qu'elle prétend combattre), mais surtout : « intimidation »: l'acte terroriste doit susciter la peur ; spectaculaire, il est conçu non seulement pour tuer mais aussi pour terrifier.

Sartre, on le voit, fait porter sur la présence de Jacques Soustelle le poids négatif $\mathrm{du}$ « politique », en soulignant le fait que le témoin de l'accusation, qui ne connaît pas l'accusé, est tout de même venu « demander sa tête 6 ». L'attaque est claire, mais elle est aussi maladroite : de fait, si la présence d'un témoin qui ne connaît pas l'accusé vient manifester que le procès est politique, alors on ne voit pas du tout en quoi la présence de Sartre, qui lui non plus ne connaît pas l'accusé, ne devrait pas être elle aussi remise en cause !

Sartre va se rendre compte rapidement de l'équivoque, et dès la deuxième version, il réécrit son départ : le mouvement part alors de sa propre ignorance, que la considération de l'ignorance de Soustelle lui a révélée et qu'il interprète tout autrement : il s'agit alors d'affirmer qu'il n'est pas venu parler politique, mais à titre d'intellectuel et d'écrivain soucieux de justice et de compréhension. Il passe ainsi de : «Soustelle ne connaît pas l'accusé » à : « Je ne connais pas Ben Sadok. »Ce déplacement, s'il disparaît comme tel de la version finale du texte (qui s'ouvre sur une considération sur la presse et le regard qu'elle porte sur le procès), n'en est pas moins essentiel à la compréhension de sa composition ; il doit être interrogé plus avant.

Cette même déclaration ouvre en effet les deux esquisses suivantes : «Je ne connais pas Ben Sadok, je ne connaissais pas Ali Chekkal, je ne puis porter témoignage sur l'Algérie ; ma déposition peut paraître inutile » (f. 1, f. 2). On la retrouvera encore dans le quatrième mouvement, lorsque Sartre rédige son plan («Je ne connais pas l'accusé, je ne connaissais pas la victime », f. 22), mais elle disparât de la version finale du texte ; autour d'elle tout un travail de composition s'effectue.

Ce double aveu d'ignorance suppose d'abord une conscience plus juste de sa position relativement à celle de Soustelle : Soustelle ne connaissait peut-être pas Ben Sadok, mais il connaissait la victime. Les journaux de l'époque, les 10 ou 11 décembre 19577, rapportent que Jacques Soustelle est venu faire « l'éloge » de la victime Ali Chekkal, qu'il avait fréquenté, notamment, dans le cadre de son mandat de gouverneur de l'Algérie, en 1955-1956. Sartre ne peut donc pas passer aisément de l'ignorance de Soustelle à la sienne, quand bien même Soustelle ne serait pas uniquement l'homme politique

6. L'intention de la formulation initiale de Sartre (« Soustelle ne connaît pas Ben Sadok ») a un double objectif : contester la double compétence (réelle ou supposée) de Soustelle, ethnologique et politique, culturelle et gestionnaire, puisque l'ethnologue spécialiste des Mayas a aussi été dans les années précédentes gouverneur de 1'Algérie. Parmi les personnalités qui viennent témoigner en faveur de Ben Sadok, Germaine Tillion présente le même profil que Soustelle : ancienne collaboratrice de celui-ci, proche de Camus, elle témoigne pourtant en faveur de Ben Sadok pour lui éviter la peine capitale. G. Tillion s'engage précisément parce qu'elle connaît l'Algérie et les Algériens, comme ethnologue spécialiste des sociétés de l'Aurès, comme éducatrice ayant effectué une mission en Algérie à l'invitation de Soustelle, et comme amie de nombreux Algériens. 7. Le Figaro du 12 décembre explique que Soustelle a protesté contre «le mythe de l'identification du FLN à l'Algérie », tout en rendant hommage à Chekkal. La Croix, le 11 décembre, cite un passage du témoignage : « C'est surtout à la session de l'ONU en janvier 1957 que j'ai apprécié son activité, son dévouement et son courage. Il a pris à l'ONU la défense des populations musulmanes contre ses prétendus libérateurs. C'est là que je vois la cause essentielle de sa mort : il portait une grave atteinte à la propagande du FLN. » 
« important » qu'il évoque ${ }^{8}$. Plus encore : même prudent, ce double aveu fait courir à Sartre le risque d'un horssujet ${ }^{9}$. « Je ne connais pas Ben Sadok » : une telle phrase, prononcée d'entrée de jeu, pourrait bien invalider le témoignage de Sartre. Il le sait d'ailleurs très bien : « ma déposition peut paraître inutile » (f. 2). Pourtant Sartre cherchera à la maintenir le plus longtemps possible ; elle permet d'en appeler à une forme objectivée de justice, et invite à un approfondissement : ne nous hâtons pas de juger, il convient peut-être de considérer les choses autrement, en cherchant à dire « toute la vérité », selon la formule que Sartre utilisa plus tard, à propos des procès de ses amis maos, alors que le dispositif judiciaire (sa façon de délimiter l'affaire) tendait à faire du procès une mascarade ${ }^{10}$.

Ce type d'accroche par affirmation d'une négation relève d'ailleurs de la rhétorique de l'incipit que Sartre affectionne. Une rapide enquête dans les textes politiques de Sartre peut être riche d'enseignement : que l'on songe à l'ouverture de la préface des Maos en France de Michèle Manceaux («Je ne suis pas mao ») ou au début de la conférence «Justice et État» («Je ne suis pas inculpé en Belgique »)11. Le sens de ces ouvertures est assez clair : il s'agit d'installer une forme d'hésitation dès les premiers temps de la déclaration, et ainsi d'inviter le lecteur à considérer les choses autrement, en tout cas à ne pas les prendre comme allant de soi ou comme « on » le dit. Ces phrases négatives visent à instiller d'emblée une prise de position à l'égard de la situation. Il ne s'agit pas de prendre parti explicitement, mais de mobiliser obliquement la puissance négatrice de la conscience du lecteur.

Certes, Sartre ne pouvait ignorer que, dans le cadre d'un tribunal, il risquait, avec une ouverture de cette forme, d'être reconduit immédiatement à une place dangereuse (celle du témoin de parti pris ou de l'intellectuel qui a un mot à dire sur tout), équivoque aussi bien politiquement que rhétoriquement. L'incipit négatif est un procédé littéraire qui engage la profondeur de sens que Sartre affectionne à l'écrit ${ }^{12}$ : il s'agit de tenir ensemble de multiples déterminations, en les donnant toutes à deviner. Mais l'intervention de Sartre au procès Ben Sadok répond à de tout autres exigences : il s'agit de sauver l'accusé de la peine de mort et, pour cela, de témoigner dans un procès politisé de toutes parts, à l'occasion duquel « on » (la presse, par exemple) réactive les divisions de la société française de plus en plus accusées en cette fin de 1957. On comprend dès lors que la formule, séduisante à l'écrit, ne figure pas dans la rédaction finale ; n'en reste qu'une trace négative, imperceptible à qui n'a pas lu le manuscrit : « J'ignore les intentions du FLN » (f. 16). Cette ouverture témoigne

8. Jacques Soustelle est un intellectuel, comme Sartre ; il a passé l'agrégation de philosophie, sortant premier de l'épreuve à 20 ans, en 1932 , après être entré premier à l'École normale supérieure, en 1929, lorsque Sartre la quittait avec les honneurs. Mais les deux hommes ont vécu différemment la seconde guerre mondiale : en séjour d'études au Mexique, Soustelle a choisi de rejoindre de Gaulle à Londres et est devenu l'un des acteurs majeurs de la contre-propagande de la France libre. À la Libération, il est élu député, puis désigné comme ministre, d'abord de l'Information, puis ministre des Colonies. Il a suivi ensuite de Gaulle dans sa retraite, tout en restant un élu de la Nation pour le RPF, avant d'être appelé fin 1954 par le président Coty à assumer le gouvernorat de l'Algérie. Alors qu'il avait des sympathies marxistes, Soustelle a donc changé de camp à l'occasion de la guerre 1940-1945. Sa position se durcira par la suite, après le retour de De Gaulle au pouvoir, dont Soustelle ne soutiendra pas la politique. Il s'engagera alors dans l'OAS et subira l'exil, de 1962 à 1968, après les accords d'Évian.

9. Le même que celui qui, par exemple, réduisit Deleuze au silence, en juin 1972, lors du procès de la mutinerie de la prison de Nancy. Voir Philippe Artières, « La prison en procès. Les mutins de Nancy (1972) », Vingtième Siècle. Revue d'histoire, n 70, 2001/2, p. 57-70, p. 69.

10. Dans la conférence « Justice et État», Sartre montre comment le tribunal transforme, jusqu'au ridicule, un procès politique en jugement d'un simple fait divers : «on me demandait de dire toute la vérité. Mais le juge voulait toute la vérité sur un incident infinitésimal : ces deux hommes étaient-ils en tel endroit ? Et nous tous nous ne pouvions comprendre qu'on n'envisage pas l'événement dans sa totalité, c'est-àdire à partir de la politique du gouvernement et du patronat. Dire toute la vérité sur un instant infinitésimal, c'est une pure contradiction. La vérité se développe dans le temps. Dans un instant borné, limité à lui-même, il n'y a pas de vérité. Mais si l'on eût rétabli la vérité, parlé de la mort des travailleurs noirs et de l'occupation du CNPF, le procès eût été politique, ce que ni le gouvernement, ni son représentant, le procureur, ni le juge ne voulaient »(«Justice et État », Situations, X, Paris, Gallimard, 1976 [1972], p. 70-71).

11. « Les maos en France », Situations, X, Paris, Gallimard, 1976 [1972], p. 38 ; « Justice et État », ibid., p. 48.

12. En 1975, Sartre définit le style comme « une manière de dire trois ou quatre choses en une » : «Il y a la phrase simple, avec son sens immédiat, et puis, dessous, simultanément, des sens différents qui s'ordonnent en profondeur. Si l'on n'est pas capable de faire rendre au langage cette pluralité de sens, ce n'est pas la peine d'écrire » («Autoportrait à soixantedix ans », ibid., p. 137). 
du fait qu'une autre difficulté est venue prendre la place de la première. Il s'agit pour Sartre de rendre impossible le déroulement d'un syllogisme politique simple : Sartre défend le FLN ; Sartre défend Ben Sadok ; donc Ben Sadok est membre du FLN. Syllogisme dont le maintien signifierait la mort pour Ben Sadok.

L'ouverture de la version finale prend justement en charge cette question :

La presse : le FLN veut faire le procès de la Guerre d'Algérie. J'ignore les intentions du FLN mais ce qui est sûr c'est qu'il ne faut pas que ce procès soit le procès de la guerre d'Algérie (f. 16).

Tout en se présentant encore devant le tribunal dans une position d'ignorance, Sartre résout le problème initial, l'effet pervers posé par le paradoxe que son incipit littéraire - relevant du régime de l'écriture et non de l'oralité - pouvait soulever. Dans le même temps se précise le cœur de la stratégie, que l'oral du témoignage devra et pourra prendre en charge en sa simplicité : l'acte meurtrier de Ben Sadok ne doit pas être confondu avec les actes terroristes du FLN.

Sartre dégage ainsi un espace pour donner sa propre lecture du choix de Ben Sadok de tuer Ali Chekkal, une lecture selon laquelle l'acte peut être compris sans que soit affirmée l'appartenance de Ben Sadok au FLN. La stratégie de Sartre est, en un sens, pratiquement impossible à tenir : témoigner, sans prendre position politiquement, d'une situation politique et historique dans laquelle l'acte de Ben Sadok prend un sens qui n'est pas le terrorisme ; s'interdire de faire de la politique, alors que (et parce que) tout le procès a manifestement une dimension politique ; ne pas prendre position relativement au FLN, bien que tout le monde connaisse sa position sur le FLN - une abstention d'autant plus exigée que la ligne de défense de Ben Sadok est qu'il a agi seul, ce qui est faux bien sûr, comme du reste tout le monde en est bien persuadé dans le prétoire, même si la police n'a alors aucune preuve de l'appartenance de Ben Sadok au FLN.

Autrement dit : Sartre doit faire du Sartre (donner une interprétation de la situation), sans faire du Sartre, en reprenant par exemple l'analyse de la situation coloniale qu'il avait présentée dans « Le colonialisme est un système » lors d'un meeting « pour la paix en Algérie » puis dans Les Temps modernes en avril 1956. Ce travail de biffure du politique se voit à plusieurs reprises dans le manuscrit, par exemple dès les premières lignes du feuillet 1 :

Je pense qu'en demandant de témoigner à un écrivain non politique, l'avocat de la défense a voulu ramener l'affaire sur son véritable plan qui est le plan de 1 à son véritable niveau qui est humain. La justice politique c'est la répression.

Cette dénégation se retrouve encore au feuillet 8 : « Maître Stibbe m'a demandé de témoigner, en dehors de toute politique. » Pourtant Sartre ne méconnaît pas que la meilleure façon de nier cette dimension politique est de la nommer pour clairement la dénoncer. C'est le sens de l'opposition entre justice politique et justice humaine, qui articule tout le témoignage. Politique signifie alors partial et renvoie à un pouvoir qui a pour but d'intimider. La justice «politique » est une justice « faussée » (comme le dit la fin de la première esquisse, f. 13) et, en cela, intimement liée au terrorisme. À l'opposé la «vraie justice » saisit l'acte commis dans sa singularité, en son lieu propre, selon une juste mesure (f. 1 : « Il faut le considérer en lui-même, cet acte »).

On l'a dit : le travail d'écriture qui se développe selon cette opposition a ceci de singulier qu'il conduit à distinguer meurtre «terroriste » et meurtre «politique », pour reconnaître, dans l'acte de Ben Sadok, un acte politique au sens le plus noble : un acte accompli au nom de la justice. Dès la première reprise du mouvement d'écriture, Sartre énumère trois déterminations, qui toutes revalorisent cette catégorie de politique : a) le meurtre politique est accompli par un solitaire (c'est un individu contre un autre), et Sartre fait fond sur des références historiques que connaît toute personne ayant appris (comme Ben Sadok) l'histoire de France à l'école : Raoul Villain assassinant Jaurès, Charlotte Corday assassinant Marat, Gavrilo Princip assassinant François-Ferdinand ; b) le meurtre politique n'a pas pour objectif de terroriser une population : il a pour but de supprimer un adversaire (qui n'est pas un ennemi), et c'est ce que Ben Sadok a fait ; c) le meurtre politique engage une conviction très grande et une solitude : on se trouve convaincu des raisons pour lesquelles on tue, et c'est dans cette conviction solitaire que l'acte a été accompli. 
$\mathrm{Au}$ fur et à mesure des réécritures, Sartre en vient à articuler de plus en plus nettement les déterminations de l'acte commis par Ben Sadok. La version finale distinguera ainsi, comme condition de l'accomplissement du meurtre politique : a) l'importance de la conviction, qui présuppose tout le long travail d'une enfance, tout un ensemble de difficultés vécues ; b) le rôle de la propagande, qui engage la prise en compte des discours et des politisations dans lesquels Ben Sadok pense son acte, et notamment les discours de la Radio du Caire ; c) le poids de la tradition, puisque le meurtrier dispose de multiples références historiques et révolutionnaires. Or cette façon de reprendre la question politique et de définir l'acte de Ben Sadok ne peut se comprendre que si l'on souligne qu'elle engage une référence constante aux positions de Camus. C'est d'ailleurs explicitement autour de Camus que Sartre va construire sa défense. On le voit dès la deuxième esquisse (f. 1), qui insiste sur le caractère non terroriste de l'acte en évoquant Les Justes (passage biffé, repris dans d'autres feuillets) : Camus a fait applaudir des terroristes au théâtre, parce qu'ils limitaient leurs actes dès lors qu'ils risquaient de tuer des innocents. Leurs objectifs étaient politiques, et non terroristes ; en outre, toute une tradition française fait l'éloge de ces actes isolés et mesurés que sont les assassinats politiques. On comprend ce qu'engage cette lecture : Ben Sadok s'inscrit dans cette tradition, il n'a rien d'un terroriste.

Comprendre la façon dont Sartre pense son intervention, c'est ainsi voir qu'il fait fond sur deux lignes directrices : d'un côté, les faits et tout d'abord la genèse de l'acte dans l'enfance même de Ben Sadok, ce qui requiert l'étude des dynamiques existentielles ; de l'autre côté, la façon dont l'acte se justifie au moins aux yeux de l'accusé, question dont l'exposé et le développement requièrent la référence à Camus et à son humanisme.

À lire Sartre, la conviction de Ben Sadok s'est forgée dans la violence : c'est sur ce fond que propagande (qui désigne le coupable) et tradition (qui autorise à le tuer) ont été reçues par l'assassin politique. Les folios 7 et 4 du manuscrit, qui s'enchaînent, ainsi que les folios 10 et 3, visent précisément à établir le sens de cette conviction, en trois temps. Le premier concerne le criminel lui-même ; le deuxième, la propagande qui l'a dirigé vers sa victime ; le troisième, le type d'acte qu'il a choisi d'accomplir.

Sartre fait d'abord remarquer que Ben Sadok avait 14 ans en 1945, lors des événements de Sétif, où l'escalade des représailles a coûté la vie à quinze mille Algériens. Il a été formé au sein du mouvement scout musulman, qui est présenté comme une «pépinière de nationalisme » (f. 713) : « Cet homme est formé par tout cela : je ne dis certes pas qu'il ait raison, je dis qu'il faut qu'il pense dans les cadres qu'on lui a imposés. » Le feuillet précise encore :

c'est à 14 ans que Ben Sadok est atteint par cela : c'est cela qui lui découvre vraiment le monde. Il avait des camarades français, il les perd. On sait qu'il rejoint les jeunes Algériens dans le mouvement scout qui est une pépinière de nationalisme. Il se forme avec des jeunes de son âge et par eux. Il appartient à une génération qui se forge au milieu de la violence. Il porte en lui l'empreinte de la violence (f. 7).

Ce n'est pas Ben Sadok qui choisit la violence, c'est la violence qui l'a obligé à choisir une communauté contre l'autre (la Nation algérienne contre la France ${ }^{14}$ ) et à s'y identifier en profondeur.

Sartre raisonne de même à propos de la relation de Ben Sadok au médium de propagande, la Radio du Caire, qui appelait à tuer Ali Chekkal. Il s'agit d'abord ici de contester l'idée que Ben Sadok aurait seulement été « influencé » par cette radio. Le problème n'est pas de savoir si la Radio du Caire est responsable de certains meurtres, le problème est que Ben Sadok était d'accord avec son message concernant la situation en Algérie : «On peut dire ce qu'on veut de la Radio du Caire. Mais ce n'est pas son procès qu'on fait ici, c'est celui d'un homme qui l'a crue parce que toute son expérience et toute son histoire le préparaient à la croire » (f. 7). Cela a

13. Cette expression est présente dans le feuillet dactylographié (f. 15). 14. Sartre éloigne ainsi la question de savoir si Ben Sadok est lié, et jusqu'à quel point, au FLN. Il s'agit ici de l'identification à une communauté nationale, pas à une organisation politique : «Rapport non à un groupe organisé mais à une communauté. Il a pu s'être trompé sur le sentiment de cette communauté, il a pu voir le problème autrement. Mais ce qui est sûr c'est que c'était elle, telle qu'il la voyait, qui était son orgueil. Comme la Serbie fut l'orgueil de Princip. Comme une certaine France fut celle de Villain ou de Charlotte Corday » (f. 3). 
une conséquence, que Sartre répète à plusieurs reprises : Ben Sadok est pleinement responsable de son acte ; il ne peut être question de demander «l'indulgence » du jury (f. 7 et 4) ; il convient simplement de veiller à rendre la justice, c'est-à-dire à juger l'acte de Ben Sadok selon la logique qu'il a suivie15.

Le troisième point concerne la tradition des assassinats politiques dans laquelle Ben Sadok ne peut manquer de penser son acte, puisqu'il a suivi les cours d'histoire de France... une histoire qui aborde bien souvent l'assassinat politique en le valorisant :

Et, justement, ne lui a-t-on pas enseigné l'héroïsme de Charlotte Corday? Ici, l'on dira : ce n'est pas la même chose. Mais la question n'est pas là. La question, c'est de savoir si lui, il pensait que c'était la même chose. Si l'exaltation de certains attentats dans l'histoire universelle ne donne pas précisément une certaine impulsion. L'attentat politique, en France, a une tradition telle que Laval luimême, blessé par Collette ${ }^{16}$, a demandé, en pleine occupation, sa grâce (f. 4).

Il est peut-être possible ici de reconnaître l'une des significations fondamentales de la formule initiale : « Je ne connais pas Ben Sadok »; elle marquait le souci de prendre ses distances à l'égard de la situation. Ce souci s'éclaire : il engage un renversement des rôles ; tout se passe comme si Sartre déclarait, en somme : «Je ne connais certes pas Ben Sadok, mais Ben Sadok me connaît et nous connaît, parce qu'il nous a étudiés, parce que son histoire et sa culture sont marquées par ce que la France lui a enseigné17. » Sartre détraque ici l'asymétrie raciste consistant toujours plus ou moins à dire que les rebelles, les fellagas, sont connus : ici, c'est le rebelle qui nous connaît.

La version finale conservera ces analyses sur l'enseignement de l'histoire de France en Algérie ; elles rendent possible la prise en compte d'un critère de distinction entre Ali Chekkal et Ben Sadok qui ne soit pas (directement) politique. Sartre va privilégier en effet, dans l'explicitation des motifs de Ben Sadok, la « différence d'âge et de génération », en repoussant les autres raisons qui pourraient être invoquées et dont il écrit précisément, dans un autre passage, qu'elles « ne comptent pas ici puisqu'elles sont politiques » (f. 10).
Les deux hommes vivent la même tragédie, ont reçu le même enseignement, mais en des temps différents ; Ben Sadok, vivant sa jeunesse dans la violence, en a retenu tout particulièrement ce qui touchait à la violence révolutionnaire :

Ali Chekkal et son assassin sont tous les deux marqués par la tragédie algérienne. Il fallait choisir pour l'un et pour l'autre. S'ils ont choisi différemment, il y a beaucoup de raisons, mais celle qui importe c'est la différence d'âge et de génération. L'un s'est formé bien avant 39 , l'autre a été marqué dès quatorze ans par la violence (f. 18).

Invoquer la jeunesse de Ben Sadok, «marqué par la violence car il a vécu tout en enfant d'abord » (f. 24), c'est pour Sartre retrouver le mouvement fondamental de sa pensée, tel qu'il venait justement de s'accentuer avec la parution de «Questions de méthode » dans Les Temps modernes en septembre et octobre 1957. La question de l'enfance y occupe une place essentielle. Sartre y évoque notamment l'enfance de Flaubert. Il écrit ainsi que ce dernier «appartient à la bourgeoisie parce qu'il est né en elle ». Il précise ensuite qu'il a fait « obscurément l'apprentissage de sa classe » à travers le prisme des contradictions d'une famille où il est le fils cadet, etc. Or Sartre insiste sur deux éléments ; d'une part, l'enfance se vit obscurément : «Le petit Flaubert a tout vécu dans les ténèbres »; et d'autre part, la signification obscure de notre existence, qui se joue dans l'enfance, est indépassable : «Tout s'est passé dans

15. À propos de la Radio du Caire, Sartre écrit : «Je ne dis pas qu'il [Ben Sadok] a eu la stupidité comme certains journaux le disent de croire cette radio. Je dis qu'il était d'accord avec ses principes et qu'il a raisonné grâce à elle, par elle » (f. 7 et 4).

16. Paul Collette a tenté d'assassiner Laval le 27 août 1941 ; il est condamné à mort le 1er octobre 1941 ; sa peine est ensuite commuée par Pétain en une condamnation à perpétuité. On peut raisonnablement soutenir que le geste de Collette a incité Sartre à écrire Les Mouches (voir Michel Contat, en collaboration avec Ingrid Galster, « Notice », Théâtre complet, Paris, Gallimard, coll. «Bibliothèque de la Pléiade », 2005, p. 1263 et 1281).

17. On peut penser que ce retournement a été suggéré à Sartre par la note dactylographiée qu'on trouve dans le dossier de la BnF (f. 15), probablement envoyée à Sartre par l'avocat de Ben Sadok, maître Stibbe. Cette note insiste sur l'attachement de Ben Sadok à la littérature française et cite notamment les noms de Sartre et Camus. 
l'enfance ». En quoi l'enfance « façonne des préjugés indépassables », autrement dit : un caractère 18 . Un des éléments du plan de Sartre résume parfaitement ce point : à propos de Ben Sadok, il précise, par distinction d'avec la conviction du terroriste, que la conviction de l'assassin politique est «aussi forte qu' un trait de caractère : formée dès l'enfance » (f. 24). « À tort ou à raison ", selon la belle et importante formule que Sartre utilisera au début de la rédaction finale de son intervention (f. 16).

Le procès Ben Sadok est ainsi l'occasion pour Sartre de mettre à l'épreuve une des thèses principales de Questions de méthode, dans un contexte où sa posture intellectuelle est devenue une question de vie et de mort. S'opère alors une série de déplacements successifs dans l'argumentation. Le premier temps consiste, on l'a dit, à soutenir que ce n'est pas Ben Sadok qui a choisi la violence, mais que c'est la violence dans laquelle il a grandi qui l'a obligé à se déterminer pour une communauté contre l'autre et à s'y identifier en profondeur. Le «monde » dans lequel Ben Sadok a grandi, «monde de la violence », est un monde où la violence n'est pas un choix parmi d'autres possibles, mais ce qui organise l'ensemble des rapports humains dans ce monde. Comment dès lors s'étonner que certains choisissent le meurtre politique?

Reste que, dans le cadre de son témoignage, Sartre ne saurait se contenter d'éclairer l'acte de Ben Sadok. Il lui faut aussi mettre en question l'attitude du tribunal qui le juge. Et c'est ici que Sartre va prendre le masque d'Albert Camus, soit le masque de celui qui se place précisément sur le plan humain, sur le plan démocratique, et s'oppose à toutes les formes de violences politiques. Et c'est ce jeu avec la figure et les thèses de Camus qui permet à Sartre de retourner le miroir vers la société qui juge.

Ce 10 décembre 1957, à Paris, Sartre joue Camus, qui a décliné l'invitation qui lui avait été faite de venir témoigner au procès, mais qui est malgré tout à la Une de l'actualité puisqu'il reçoit ce même jour à Stockholm le prix Nobel de littérature. Cette absence est providentielle ; brouillé avec Camus depuis plusieurs années, Sartre décide de confondre un temps leurs deux œuvres et leurs biographies, de dissimuler leur brouille par une stratégie de brouillage. Il y a ici de nombreuses ironies... on rappellera que l' « amalgame » était le motif de la rupture entre les deux hommes en 195219 ; dans La Chute, en 1956, Camus avait répondu à la critique virulente de Sartre en faisant de l'《amalgame » la «méthode » de son personnage principal, Jean-Baptiste Clamence : il s'agit de « défendre le voleur en faisant valoir les crimes de l'honnête homme 20 ». Sartre a apprécié le livre - «le meilleur de Camus » selon lui21 - c'est peut-être à cause de la façon dont Camus reprenait à son compte le terme d' « amalgame »; il semble en tout cas que Sartre utilise la même méthode, lors de sa déposition au procès Ben Sadok, pour entrer dans la peau de Camus afin de sauver celle de Ben Sadok.

Jouer à être Camus permet par ailleurs à Sartre de faire face à de multiples contraintes. Il s'agit de ne pas politiser à outrance un procès qui risque bien de l'être déjà beaucoup trop ; or justement Camus ne veut pas prendre position. Il s'agit de ne pas méconnaître la situation vécue de Ben Sadok ; or Camus est justement né en Algérie. Il s'agit, plus encore, de mobiliser la méthode de l'amalgame pour dénoncer un autre amalgame : la confusion entre assassinat politique

18. Questions de méthode, dans Critique de la Raison dialectique, précédé de Questions de méthode, Paris, Gallimard, 1985, citations p. 54-56.

19. Voir « Réponse à Albert Camus » (1952), dans Situations, IV, Paris, Gallimard, 1964, p. 101-102.

20. A. Camus, La Chute, Paris, Gallimard, coll. « Folioplus », 2008, p. 77-78. Il ne serait sans doute pas impossible de montrer qu'au fond Clamence, c'est Camus et Sartre en même temps. Plusieurs commentateurs ont noté que Camus s'approprie ainsi le reproche d'amalgame que Sartre lui avait fait au moment de la brouille avec Les Temps modernes (voir Gilles Philippe, « Notice » de La Chute, dans A. Camus, Euvres complètes, t. III, Paris, Gallimard, coll. «Bibliothèque de la Pléiade », 2008, p. 1368 ; Jean-Louis Prat, «Sartre, Camus, Clamence », Revue du MAUSS permanente, 29 octobre 2011 [en ligne], <www.journaldumauss. net/spip.php?article842>).

21. Selon un témoignage répété d'Olivier Todd, Sartre a précisément apprécié La Chute parce que Camus «s'y est mis et caché tout entier » (« Il faut garder Camus vivant. Il permet de réfléchir », Le Monde, 20 novembre 2009). 
et terrorisme. Dans le plan, puis par deux fois dans la rédaction finale, à son début puis à la fin, Sartre résume ainsi son propos en disant qu'il faut «éviter les amalgames », éviter l'amalgame entre la guerre d'Algérie et le procès Ben Sadok (f. 22) ; éviter l'amalgame, "distinguer clairement et en dehors de toute prise de position sur la guerre » (f. 16), entre terrorisme et assassinat politique ; éviter l'amalgame enfin entre justice politique et justice humaine (f. 20).

En écrivant Les Justes, qui loue les anarchistes russes qui préfèrent renoncer à leur projet plutôt que de risquer de tuer un innocent, Camus savait certes que la situation avait changé, que 1950 n'était plus 1905 ; Sartre s'ingénie à retourner le geste de Camus, pour montrer qu'en 1957 encore un homme, Mohamed Ben Sadok, a pu se hisser au niveau de Kaliayev en éprouvant «les efforts démesurés [qui sont nécessaires] pour se mettre en accord avec le meurtre », pour lesquels Camus dit son « respect» et son « admiration », ainsi que sa «fidélité 22 ». Se référer à cette pièce, c'est donc pour Sartre inscrire l'acte de Ben Sadok dans une tradition française et politique, et ainsi réaliser une seconde politisation « indirecte » de la situation - l'évocation de l'enfance constituant la première - qui a pour but de retourner l'acte meurtrier de Ben Sadok contre la société qui l'accuse. Qui a donné à Ben Sadok les moyens de réaliser sa révolte? se demande Sartre à plusieurs reprises. C'est « nous » : «Et Ben Sadok ? D'où ont pu lui venir la tradition et les exemples ? Je le dis nettement : de la France et de son enseignement » (f. 11). Cela donne dans la rédaction finale :

Mais si du moins on pourait avait pu lui dire : l'assassinat politique inspire l'horreur partout. Or il y a une tradition de l'assassinat politique. Charlotte Corday 23 , nous le savons, pensait à Brutus qui tua César et à tous les Romains dont on lui avait parlé et qui avaient abattu des tyrans. Et chez lui, chez Ben Saddok, de (sic) où vint cette tradition : de nous. C'est dans les manuels d'histoire utilisés en Algérie et rédigés en France qu'on peut voir cela. Charlotte Corday est une héroïne nationale : on le lui a appris. Ben Sadok n'est pas Charlotte Corday ; c'est bien possible mais l'essentiel c'est qu'on lui a appris à croire qu'il pourrait l'être (f. 19).
L'autre intérêt de la référence à Camus tient aux positions de celui-ci sur la peine de mort. De fait, au moment du procès, nul n'ignore que Camus a publié des «Réflexions sur la guillotine », dans un volume cosigné avec Arthur Kœstler, Réflexions sur la peine capitale. Au cœur de ce livre se trouve une interrogation profonde sur la fonction d'intimidation qu'endosse la peine de mort. Pour Sartre, ce motif prend une signification déterminée : faire prévaloir une justice humaine, qui ne tient compte que de l'acte, sur une justice politique qui chercherait précisément à « intimider » les indépendantistes algériens. Dans l'ensemble du manuscrit, le vocabulaire de l'intimidation est très fréquent. Et, dans la version finale, c'est sur ce thème que Sartre choisit de conclure son intervention :

[...] Appliquer la loi à un homme qui a tué pour ce qu'il a fait sans songer à rien d'autre qu'à son acte, c'est rendre justice. La répression commence lorsqu'on pense aux répercussions que la sentence peut avoir ailleurs, lorsqu'on condamne un homme vivant, bien réel, en pensant d'abord à intimider les autres (f. 20).

Dans son texte, Camus s'interroge sur le sens qu'il y a à maintenir l'exécution capitale dans l'obscurité d'une cour de prison au petit matin, alors que le sens de cette peine a justement été de «prévenir », d'empêcher

22. Nous citons ici la présentation des Justes par Camus lui-même (Paris, Gallimard, coll. «Folio », 1999, p. 7). En réalité, pour Sartre, l'opération n'est pas neuve. Il l'a déjà accomplie en écrivant Les Mains sales, en même temps que Camus écrivait Les Justes. Sandra Teroni a bien montré les jeux de «masques » entre les deux pièces. Celle de Sartre contient d'ailleurs un résumé de l'intrigue de celle de Camus. Au-delà des jeux intertextuels habituels, S. Teroni parle ici de deux « créations parallèles » (« Notice » des Mains sales, dans Théâtre complet, op. cit., p. 1377).

23. Cette référence à Charlotte Corday étonna Georges Sinclair qui, dans son article de Combat du 11 décembre 1957, rappela que le meurtre politique l'a conduite à la peine capitale. L'exemple de Charlotte Corday ne vient pas de Camus, mais probablement de Pierre Drieu la Rochelle qui lui avait consacré une pièce en 1939 (Charlotte Corday, Paris, Gallimard, 1944). Cette pièce constitue d'évidence un pré-texte de celles de Sartre et de Camus. 
d'autres crimes, bref d' « intimider24 ». Pour lui, si elle n'est plus capable d'intimider, si on ne peut plus supporter de la regarder en face, la peine de mort n'est plus que vengeance. Camus poursuit sa critique en soulignant un second archaïsme de la peine de mort, qui n'a de sens qu'à pouvoir être « rattrapée » par une justice divine. La peine de mort est une «peine religieuse $25 »$. Hors de cette croyance, elle soumet ses victimes aux aléas des temps et des lieux : on tue aujourd'hui un ouvrier communiste français en Algérie parce qu'on a condamné à mort des Algériens, parce que la justice française veut montrer qu'elle ne traite pas de façon différente un Français et un Arabe ; en d'autres circonstances, on ne l'aurait pas tué26. La peine de mort est désormais une peine «politique », bien plus qu'une peine qui condamne des crimes de droit commun, au point, écrit Camus, que « chacun d'entre nous, si honorable soit-il, peut envisager la possibilité d'être un jour condamné à mort27 ».

Sartre avait lui aussi pris la mesure de ce qui se jouait dans le procès pour Mohamed Ben Sadok : pour défendre un homme qui risque sa tête, il faut trouver le bon moyen de mettre le tribunal face à ses responsabilités et face à ses possibles contradictions. C'est pourquoi il cherche non seulement à requalifier l'acte de Ben Sadok (assassinat politique et non crime terroriste), mais aussi à amener la Cour à réfléchir sur ses propres actes. La thématique de l'intimidation autorise ce passage de la première perspective à la seconde. La suspicion de pratiquer la terreur ne vise plus l'accusé, mais bien le tribunal qui juge28.

Lorsqu'on lit la presse du 11 décembre 1957, on peut se demander si le passage de l'écrit à l'oral n'a pas finalement effacé le nom même de Camus du témoignage de Sartre : les articles sur le procès ne précisent jamais que Sartre ait fait référence à Camus. En un sens, l'élision du nom de Camus confirmerait paradoxalement la stratégie de Sartre : prendre le masque de Camus exigeait, peut-être, que le nom même du masque, qui a guidé jusque-là les rédactions successives du texte, disparaisse du témoignage oral que Sartre livre à la Cour. Reste que l'on est ici dans l'incertitude.

Ce soupçon d'un silence rend la lecture du manuscrit encore plus singulière : préparer une intervention orale est une chose, la prononcer en est une autre, et le fait que l'on n'ait aucun enregistrement de ce que Sartre a dit lors du procès fait considérer le texte préparatoire comme un ensemble encore lacunaire, indécidable en un sens. Une chose reste certaine, que le témoignage de Simone de Beauvoir laissait déjà entendre : cette intervention a imposé à Sartre de faire ce qu'il ne fit que

24. Voir notamment, A. Camus et A. Kœstler, Réflexions sur la peine capitale, Paris, Calmann-Lévy, 1957, p. 125, 128, 130, 132, 135, 139.

25. A. Camus, « Réflexions sur la guillotine», dans Réflexions sur la peine capitale, op. cit., p. 161.

26. Ibid., p. 155 : «Les hasards du temps rejoignent ceux de la géographie pour renforcer l'absurdité générale. L'ouvrier communiste français qui vient d'être guillotiné en Algérie pour avoir déposé une bombe (découverte avant qu'elle n'explose) dans le vestiaire d'une usine, a été condamné autant pour son acte que pour l'air du temps. Dans le climat actuel de l'Algérie, on a voulu à la fois prouver à l'opinion arabe que la guillotine était faite aussi pour les Français et donner satisfaction à l'opinion française indignée par les crimes du terrorisme. »

27. Ibid., p. 165. Il est tout à fait frappant de remarquer qu'au moment de sa mort Camus était en train de rédiger un récit largement autobiographique (Le Premier Homme), dont les notes préparatoires présentent des esquisses de dialogue entre Cormery-Camus et un ami algérien, qui est comme un frère pour lui, «Saddok », dont le nom hante véritablement les notes et les plans préparatoires du livre. Saddok est pris entre la France et l'Algérie, entre son affection pour sa « famille » française et la nécessité d'adopter les traditions algériennes, non pas parce qu'il adhère à ces valeurs mais parce que l'histoire y a figé son peuple. Dans ces remarques, Camus consent à comprendre très profondément la situation d'un homme comme Mohamed Ben Sadok : «Les deux nationalismes algériens. L'Algérie entre 39 et 54 (rébellion). Ce que deviennent les valeurs françaises dans une conscience algérienne, celle du premier homme. La chronique de deux générations explique le drame actuel »(Euvres complètes, op. cit., t. IV, p. 941). D'une certaine façon, la boucle est bouclée ; Camus a rejoint Ben Sadok ; la mort de Camus laissera pourtant cet ultime « amalgame » dans l'ombre. 28. À cet égard, il s'agit peut-être de se rappeler que pendant l'été 1957 l'avocat Jacques Vergès avait choisi de mettre en œuvre pour la première fois la stratégie du «procès de rupture » dans un autre procès de la guerre d'Algérie. Un livre en est tiré qui paraît en novembre aux Éditions de Minuit, Pour Djamila Bouhired. Sartre ne pouvait certes pas se permettre ce genre d'expérimentation dans le procès Ben Sadok. Un autre événement de l'automne 1957 a peut-être lancé la rédaction de Sartre. Il concernait aussi la question de la peine de mort. Il s'agit d'un film sur l'affaire Eugène Weidmann qui a été diffusé par 1'ORTF le 22 octobre, avec le jeune Laurent Terzieff dans le rôle-titre. Tueur en série d'origine allemande, Weidmann fut en 1939 le dernier condamné à mort guillotiné en public en France ; Sartre cite son nom dans Saint Genet, comédien et martyr (Paris, Gallimard, 1952). 
rarement, parler sous contrainte en acceptant de modérer et même de travestir la radicalité de son engagement. Pestant contre la stratégie que le cadre du procès lui imposait, embarrassé d'être ce témoin ne connaissant pas l'accusé et d'avoir à taire ce qu'il pensait du caractère inévitable du terrorisme, Sartre a dû, tout en témoignant, retrouver et éprouver dans un vif inconfort quelques-unes des nombreuses contradictions qu'il eut à affronter à chaque moment de sa vie publique. De ces tensions, qui sont la nécessaire réalité de l'engagement, la rédaction des quelques feuillets que conserve la $\mathrm{BnF}$ portent encore les traces ; elles n'ont pas fini de susciter nos interrogations. 
Jean Bourgault est professeur de philosophie au lycée Condorcet (classe de Première supérieure). Docteur en philosophie, auteur de nombreux articles sur Sartre, il a coanimé l'équipe Sartre de l'ITEM (CNRS-ENS) avec Gilles Philippe durant sept années. Il est membre du comité de rédaction des Temps modernes et de celui des Études sartriennes

jnbrglt@gmail.com

Grégory Cormann enseigne la philosophie sociale à l'Université de Liège où il codirige également l'Unité de recherches en philosophie politique. Il est l'auteur d'un grand nombre d'articles portant sur les rapports entre phénoménologie et sciences sociales. Il est membre de l'équipe Sartre de l'ITEM (CNRS-ENS) et du CA du Groupe d'études sartriennes international. Son ouvrage à paraître, Sartre, les passions de la liberté. Phénoménologie, politique, savoirs critiques (Peter Lang, 2014), pose les fondements d'une anthropologie politique des émotions.

gregory.cormann@ulg.ac.be

\section{Résumés Quelques remarques sur le témoignage de Sartre au procès Ben Sadok}

Le 10 décembre 1957, Sartre est appelé à témoigner en faveur de Mohamed Ben Sadok, qui avait assassiné Ali Chekkal, ancien viceprésident de l'Assemblée algérienne : la défense veut tout faire pour que Ben Sadok ne soit pas condamné à mort. On étudie ici, à partir du manuscrit, la genèse et le sens du témoignage de Sartre. Sartre est dans une position paradoxale : il ne peut soutenir publiquement le terrorisme du FLN, alors qu'il l'approuve ; en pleine guerre d'Algérie, il lui faut donc en appeler à la justice en tenant à distance la question politique. Sartre le fait en explicitant la difficile jeunesse de Ben Sadok, puis en évoquant les célèbres assassinats politiques de l'histoire de France, dont on a souvent fait l'éloge, enfin en se référant à l'humanisme de Camus. Sartre est prêt à jouer tous les rôles pour sauver Ben Sadok de la peine de mort.

On December 10th 1957, Sartre was called to the witness-box in support of Mohamed Ben Sadok, who had assassinated Ali Chekkal, the former Vice-Chairman of the Algerian Assembly. The defense was trying to avoid a death sentence for Sadok. On the basis of the manuscripts, we have studied the elaboration and the meaning of Sartre's evidence in favour of Sadok. Sartre found himself in a paradoxical position: although he approved of it, he could not publicly support the National Liberation Front's terrorist line of action. Right in the midst of the Algerian war, he then had to invoke justice, while standing completely clear of the political question. Sartre did so by dwelling on Ben Sadok's difficult youth, by bringing up the famous political assassinations in French history, often the object of praise, and finally by referring to Camus's humanism. Sartre was ready to play any role to save Ben Sadok from the death penalty.

Am 10. Dezember 1957 wird Sartre eingeladen, zugunsten des Angeklagten Ben Sadok, Mörder des ehemaligen Vize-
Präsidenten des algerischen Parlaments Ali Chekkal, als Zeuge auszusagen. Die Verteidigung Ben Sadoks will um jeden Preis den Angeklagten vor der Todesstrafe retten. Ausgehend von Sartres Manuskript, versucht dieser Text Genese und Sinn seiner Aussage zu ermitteln. Sartre befindet sich in einer paradoxalen Position: Er kann den FLN-Terrorismus öffentlich nicht befürworten, obwohl er ihn als legitim betrachtet. Im Kontext des Algerien-Krieges muss er deshalb die politische Frage fern halten und sein Plädoyer ganz auf die Frage der Gerechtigkeit konzentrieren. Dies schafft Sartre, indem er zuerst Ben Sadoks schwierige Jugend erwähnt, dann die berühmten politischen Morde der französischen Geschichte, die oft gelobt oder nachträglich legitimiert wurden, schließlich, indem er sich auf Camus' Humanismus bezieht. Sartre scheint also bereit, verschiedene Positionen einzunehmen, um Ben Sadok vor dem Todesurteil zu retten.

E1 10 de diciembre de 1957, Jean Paul Sartre es convocado a testimoniar a favor de Mohamed Ben Sadok, quien había asesinado a Alí Chekkal, ex vicepresidente del Congreso argelino; la defensa está dispuesta a todo para que Ben Sadok no sea condenado a muerte. Estudiaremos aquí, a partir del manuscrito, la génesis y el sentido del testimonio de Sartre. Sartre se encuentra en una posición paradójica: no puede sostener públicamente el terrorismo del FNL, aun cuando lo aprueba; en plena guerra de Argelia, no puede sino apelar a la justicia, manteniendo a distancia la cuestión política: lo hace explicitando la juventud difícil de Ben Sadok, evocando luego asesinatos políticos célebres de la historia de Francia, algunos de los cuales han sido objeto de elogios, para referirse finalmente al humanismo de Camus. Sartre está decidido a jugar todos los papeles para salvar a Ben Sadok de la pena de muerte. 
Em 10 de dezembro de 1957, Sartre depõe em favor de Mohamed Ben Sadok, que tinha assassinado Ali Chekkal, antigo vice-presidente da Assembleia argelina. A defesa queria evitar que Ben Sadok fosse condenado à morte. Examina-se aqui, a partir do manuscrito, a génese e o significado do testemunho de Sartre, que se encontrava em posição paradoxal: não podia apoiar publicamente o terrorismo da FLN, ainda que o aprovasse; em plena guerra da Argélia, tinha de apelar à justiça mantendo a questão política à distância. Para tal, retraça a difícil juventude de Ben Sadok, evoca célebres assassinatos políticos da história da França, muitas vezes louvados, e finalmente socorre-se do humanismo de Camus. Sartre presta-se a desempenhar todos esses papéis para salvar Ben Sadok da pena de morte.
I1 10 dicembre 1957, Sartre è chiamato a deporre dalla difesa di Mohamed Ben Sadok, accusato dell'omicidio di Ali Chekkal, ex-vicepresidente del Parlamento algerino: la difesa vuole fare tutto il possibile affinché Ben Sadok non sia condannato a morte. In questo testo vengono studiati, a partire dal manoscritto, la genesi e il senso della testimonianza di Sartre. Sartre è in una posizione paradossale: non può sostenere pubblicamente il terrorismo del FLN, che tuttavia approva; in piena guerra d'Algeria, deve fare appello alla giustizia restando a distanza dalle questioni politiche. Sartre spiega dunque la giovinezza difficile di Ben Sadok, quindi evoca i celebri omicidi politici della storia francese, considerati come momenti fondatori, e infine si richiama all'umanesimo di Camus. Sartre è pronto a interpretare tutti i ruoli possibili per salvare Ben Sadok dalla pena di morte. 